\title{
Performing Gamma Knife radiosurgery safely during the COVID-19 pandemic: preliminary results from a single center in the Lombardy region in Italy
}

\author{
Andrea Franzini ${ }^{1} \cdot$ Luca Attuati $^{1} \cdot$ Maurizio Fornari $^{1} \cdot$ Franco Servadei $^{1,2} \cdot$ Pierina Navarria $^{3} \cdot$ Piero Picozzi $^{1}$
}

Received: 30 April 2020 / Accepted: 6 May 2020 / Published online: 18 May 2020

(C) Springer-Verlag GmbH Austria, part of Springer Nature 2020

Keywords COVID-19 $\cdot$ Gamma knife $\cdot$ Radiosurgery $\cdot$ Brain metastasis $\cdot$ Lombardy $\cdot$ Italy

We read with interest the editorial of Zoia and colleagues about the reorganization of neurosurgical activities to face the COVID-19 outbreak.[4] However, at this time, there are no best practice recommendations for cranial radiosurgery in the setting of COVID-19.[3] Based on our experience, the aim of this letter is to describe a safe treatment pathway for patients in need of cranial radiosurgery during the COVID-19 pandemic.

Since late February 2020, hospitals in Lombardy, Italy, began admitting an increasing number of patients with COVID-19. Elective surgeries were halted, and operating rooms were converted into COVID-19-dedicated intensive care units. In mid-March, neurosurgical elective activity ceased, and oncological and traumatic cases were then referred to dedicated hub hospitals. [1]

Our institution is a general hospital and is outfitted with a Gamma Knife (GK) unit. We are the closest (about $40 \mathrm{~km}$ ) to the epicenter of the SARS-CoV-2 outbreak in Italy (Codogno, Lombardy). As such, we rapidly converted our facility into a hospital largely for COVID-19 patients. After this, we had to face two contrasting needs for patients who required cranial radiosurgery. On one hand, we had to treat patients with urgent

This article is part of the Topical Collection on Functional Neurosurgery Other

Andrea Franzini

andrea.franzini1@ hotmail.it

1 Department of Neurosurgery, Humanitas Clinical and Research Center - IRCCS, via Manzoni 56, 20089 Milan, Rozzano, Italy

2 Department of Biomedical Sciences, Humanitas University, Via Rita Levi Montalcini 4, 20090 Milan, Pieve Emanuele, Italy

3 Department of Radiation Oncology, Humanitas Clinical and Research Center - IRCCS, Milan, Rozzano, Italy intracranial pathology.[3] Notably, patients with brain metastases are at risk, as a delay in treatment would affect their longterm prognoses. On the other hand, we had to prevent our patients from nosocomial infection with COVID-19 that could become life-threatening.[2] There was a significant risk to these patients as our hospital was filled with over 300 patients being treated for COVID-19, as well as many healthcare workers who were likely carrying the virus while asymptomatic. Therefore, we decided to treat those patients by utilizing a treatment pathway that was still able to ensure patient safety.

Some precautions were used. Only one patient was treated per day (usually two patients are treated with GK radiosurgery per day at our center). All healthcare workers who came into contact with the patient, including the treating neurosurgeon and nurses, were excluded from treating patients with COVID-19 and from being in COVID-19 units. These workers and patients also wore appropriate personal protective equipment, including gloves and facemasks, during all steps of the GK procedure.

Our treatment pathway for GK radiosurgery was performed as an outpatient procedure that spanned 2 days. During the first day, the patient underwent COVID-19 nasopharyngeal swab, chest-computed tomography (CT), routine blood tests, and an infrared body temperature check. The second day, if all those exams and physical examination had ruled out COVID19 infection and related syndromes, the patient was admitted to the GK center. After arrival, the patient washed his/her hands with hand sanitizer and stored in a locker all personal belongings. Peripheral pulse oximetry was placed to assess oxygenation status, and the stereotactic head frame was fixed. The patient was transferred to radiology procedural suit and back by traveling on a wheelchair. Stereotactic magnetic resonance imaging was acquired, and radiosurgery was performed with the Leksell GK Perfexion (Elekta AB instruments, Stockholm, Sweden). At the end of the procedure, 
the frame was removed, and the patient was discharged home. Finally, the GK and radiology rooms were sanitized and disinfected carefully.

Since the beginning of the outbreak in late February 2020, we have treated 12 patients with single or multiple brain metastases at our GK unit (seven new and five repeat treatments). At the end of April 2020, the authors confirmed via telephone interview that none of these patients developed COVID-19 pneumonia and that all were still alive. The number of patients treated has been considerably reduced during the pandemic than routinely, as an average number of 40 patients is usually treated with GK radiosurgery per month at our center.

Several aspects favor GK radiosurgery in the current pandemic setting. It can be performed in outpatient setting as a single-session procedure, opposite to conventionally fractionated radiotherapy, which requires between 10 and 30 hospital visits. Second, GK radiosurgery does not require endotracheal intubation or the use of high-speed instruments, lowering the risk of exposure to infectious aerosols and bone dust present in open surgical procedures.[2] Treating patients at their oncological reference center instead of being referred to distant centers is also relevant. As an example, we are able to make direct online comparison between a patient's latest brain imaging and stored exams/plans using the Leksell GammaPlan software (Elekta AB instruments, Stockholm, Sweden). This allows to distinguish new metastatic lesions from remnants of previous treatments or presumed radiation necrosis, as well as to make more accurate dosimetric prescriptions together with our radiotherapists and medical physicists. Finally, patients can be directly assessed by their personal oncologist while at their reference center.

Our protocol for performing GK radiosurgery during the COVID-19 outbreak has demonstrated to be safe and could be extended to other outpatient neurosurgical procedures. Notably, this treatment pathway could be adopted by other
GK units working in the vicinity of a COVID-19 outbreak without having to transfer patients to distant centers.

Acknowledgments The authors thank Dr. Shayan Moosa, MD (University of Virginia, Charlottesville, Virginia, USA) for the English language edits.

\section{Conflict of interest}

The authors have no personal, financial, or institutional interest in any of the drugs, materials, or devices described in this article.

Informed consent The patients have consented to submission of this letter to the journal.

Abbreviations GK, gamma knife; CT, computed tomography

\section{References}

1. Cenzato M, DiMeco F, Fontanella M, Locatelli D, Servadei F (2020) Editorial. Neurosurgery in the storm of COVID-19: suggestions from the Lombardy region, Italy (ex malo bonum). J Neurosurg 1-2. https://doi.org/10.3171/2020.3.JNS20960

2. Grasselli G, Zangrillo A, Zanella A, Antonelli M, Cabrini L, Castelli A, Cereda D, Coluccello A, Foti G, Fumagalli R, Iotti G, Latronico N, Lorini L, Merler S, Natalini G, Piatti A, Ranieri MV, Scandroglio AM, Storti E, Cecconi M, Pesenti A, Network C-LI (2020) Baseline Characteristics and outcomes of 1591 patients infected with sarsCoV-2 admitted to ICUs of the Lombardy Region, Italy. JAMA. https://doi.org/10.1001/jama.2020.5394

3. Liaw J, Patel VA, Bann DV, Saadi RA, Mau C, Brettler S, Tuanquin L, Zacharia BE, Isildak H (2020) Letter: COVID-19 pandemic: safety precautions for stereotactic radiosurgery. neurosurgery. https://doi. org/10.1093/neuros/nyaa163

4. Zoia C, Bongetta D, Veiceschi P, Cenzato M, Di Meco F, Locatelli D, Boeris D, Fontanella MM (2020) Neurosurgery during the COVID19 pandemic: update from Lombardy, northern Italy. Acta Neurochir (Wien). https://doi.org/10.1007/s00701-020-04305-w

Publisher's note Springer Nature remains neutral with regard to jurisdictional claims in published maps and institutional affiliations. 